\title{
Nuevas evidencias de infraestructura asociada al sistema hidráulico del complejo arqueológico de Tunanmarca, Jauja
}

\author{
New evidences of infraestructure associated with hydraulic system of the archaeological \\ complex of Tunanmarca, Jauja
}

\author{
Manuel F. Perales Munguía ', Henoch Loayza Espejo ${ }^{2}$ \\ Universidad Continental
}

\section{RESUMEN}

Objetivos: Identificar evidencias que prueben la asociación cronológica entre el asentamiento prehispánico de Tunanmarca (Jauja - Junín) y el sistema hidráulico cercano a éste; y evaluar el papel de dicha asociación y del manejo del agua en la constitución del poder en la jefatura con sede en dicho asentamiento. Métodos: Se desarrolló esta investigación básica, con un alcance descriptivo y diseño transversal. Los procedimientos de recolección de datos estuvieron basados en: reconocimiento pedestre a nivel de superficie, en transectos; para la ubicación y registro de las evidencias se empleó un GPS, brújula, cintas métricas, nivel y cámara fotográfica, en tanto que los datos espaciales fueron procesados en el software ArcGIS y otros. Resultados: Se identificaron los restos de tres caminos prehispánicos, además de dos estructuras arqueológicas asociadas al sistema hidráulico de Tunanmarca, una de las cuales parece haber sido un reservorio de agua. El camino más largo une el probable reservorio y el tramo final del canal con el asentamiento de Tunanmarca. Conclusiones: El asentamiento prehispánico de Tunanmarca se asocia cronológicamente con el sistema hidráulico cercano, principalmente con el tramo final del canal principal, que parece terminar en un probable reservorio de agua, al que se accede desde el asentamiento mediante un camino prehispánico. Asimismo, la escasa sofisticación de la tecnología hidráulica observada y la presencia del camino que une la infraestructura asociada al canal con la parte central del sitio de Tunanmarca,

1 Bach. en Ciencias Sociales y Licenciado en Arqueología, Estudios de Maestría en Antropología Jurídica. Docente de la Universidad Continental.

2 Lic. en Educación, Docente cesante del I. S. P. “Pedro Monge Córdova” de Jauja. 


\section{ABSTRACT}

Objectives: To identify evidences that prove chronological association between the pre hispanic settlement of Tunanmarca (JaujaJunín) with the hydraulic system near to it; and evaluate the role of such association and water management on the constitution of the power in the chiefdom built around this settlement. Methods: This basic investigation was developed, with a descriptive scope and transversal design. The procedures of compilation of data were based on: pedestrian recognition of surface leve; transects to locate and record evidences, was employed a Global Positioning System (GPS); compass tape measure, gradienter and photographic camera. While the spatial data was processed in software ArcGIS. Results: Three prehispanic remains of roads were identified, besides of two archeological structures associated to the hydraulic system of Tunanmarca, one of which seems to have been a water reservoir. The longer road connects to a probable reservoir and to the final stretch of the canal with the settlement of Tunanmarca. Conclusions: The prehispanic settlement of Tunanmarca is chronologically associated with the hydraulic system near to it, principally with the final stretch of the principal water canal, which seems to end in a possible reservoir of water, to which one accedes from the settlement by a prehispanic road. Also the limited sophistication in hydraulic technology observed and the presence of the road that connects the infrastructure associated with the canal with the central part of Tunanmarca, suggest some management water importance in the politic power constitution process and in the authority exercise of the prehispanic chief of Tunanmarca.

Key words: Infrastructure, hydraulic system, archaeological complex, Tunanmarca, Jauja.

\section{INTRODUCCIÓN}

Una de las grandes preocupaciones de la antropología ha sido el tema del origen del gobierno $(1,2,3,4)$ y con él, la cuestión sobre la naturaleza del poder y su papel en los procesos que condujeron al surgimiento de los sistemas políticos complejos (5). En este contexto, diversos estudios han logrado identificar un tipo de organización sociopolítica de alcance regional, denominado jefatura, que se caracteriza por contar con formas relativamente institucionalizadas de gobierno y algún nivel de estratificación social, cuyos miembros tienen una economía basada en la producción intensiva de alimentos, así como

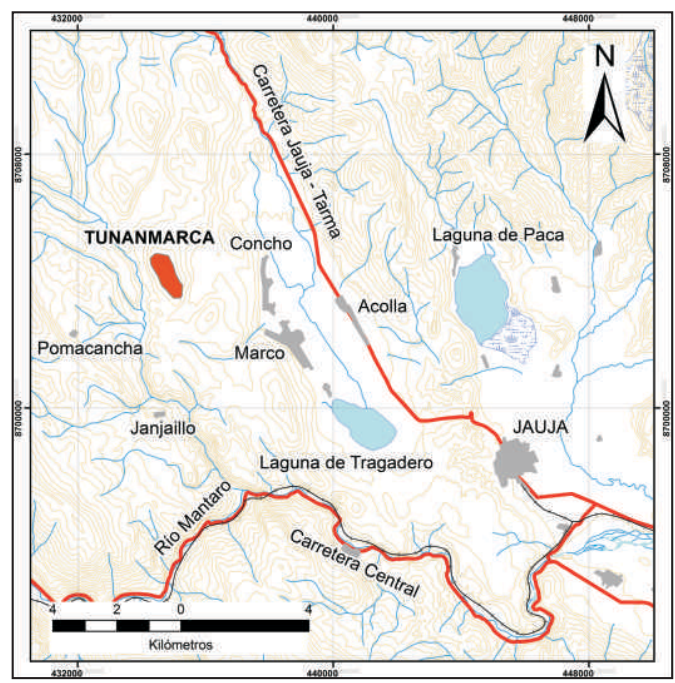

Figura 1: Mapa de ubicación del complejo arqueológico de Tunanmarca (basado en la Carta Nacional IGN, hojas 24-I y 24-m, esc. 1:100,000. Intervalo de grilla: 8,000 m; esc. de publicación: 1:100,000)

una estructura social fuertemente establecida sobre las relaciones de parentesco, las mismas que suelen propiciar un acceso diferencial a los recursos para la subsistencia $(6,7,8)$.

Los estudios llevados a cabo en Jauja entre las décadas de 1970 y 1980 por el Upper Mantaro Archaeological Research Project (UMARP) de la Universidad de California en Los Angeles (UCLA), específicamente en el valle de Yanamarca, han señalado que las 
diversas comunidades prehispánicas que ocuparon dicho territorio durante la segunda mitad del periodo Intermedio Tardío (ca. 1350-1450 d.C.) estuvieron organizadas precisamente bajo la forma de jefaturas $(7,9,10)$, entre las cuales habría destacado una que tuvo su sede en el extenso asentamiento de Tunanmarca, la cual incluyó a los habitantes de otros pueblos vecinos como Umpamalca y Chahuín (7). Según algunos documentos coloniales tempranos $(11,12)$, en dicha organización sociopolítica y en otras similares de la región, el poder, inestable aún, estuvo en manos de ciertos líderes denominados sinches o cinchecona, valerosos en la guerra y hábiles para defender los intereses de la comunidad. Esto, sumado a la evidencia recuperada por la arqueología, ha llevado a algunos estudiosos a plantear que la principal base de la autoridad que ejercieron los líderes en las jefaturas del valle de Yanamarca fue el poder militar, dejando a un segundo plano el poder económico y el poder ideológico. (7)

Un tipo de evidencia arqueológica que resulta importante para el estudio del papel del poder económico en la consolidación de la autoridad de los líderes de una jefatura es la infraestructura asociada a la intensificación de la producción agropecuaria y el manejo de recursos clave como el agua (7). Precisamente, en el caso de Tunanmarca, contamos con los restos de un interesante sistema hidráulico asociado a dicho asentamiento, el mismo que fue reportado inicialmente por Jeffrey Parsons en la década de 1970 (13) y fue estudiado con alguna profundidad más tarde por el proyecto UMARP (14). No obstante la importancia de estos trabajos, no se contaba con evidencia suficiente que permitiese probar la asociación directa entre dicho sistema hidráulico y el sitio de Tunanmarca.

Por ello, nuestra investigación se orientó principalmente a encontrar tal evidencia, además de evaluar el papel que pudo haber jugado el manejo del agua en la construcción y la consolidación de la autoridad de los líderes de la jefatura de Tunanmarca y, de este modo, contribuir en el estudio de la evolución sociopolítica de los pueblos prehispánicos del valle de Yanamarca. Para alcanzar estos objetivos llevamos a cabo un reconocimiento arqueológico a nivel de superficie en las áreas ubicadas entre el tramo final del canal principal del sistema hidráulico de Tunanmarca y el asentamiento del mismo nombre (Figura 01).

\section{MATERIAL Y MÉTODOS}

El reconocimiento del terreno se hizo íntegramente a pie, durante el mes de febrero de 2011, siguiendo los indicios identificados previamente por el segundo autor y con la asistencia de dos ayudantes, quienes se desplazaron de forma paralela y perpendicular al trazo y ubicación de los distintos rasgos arqueológicos estudiados, siguiendo el criterio de transectos, cuya utilidad ha sido ampliamente demostrada

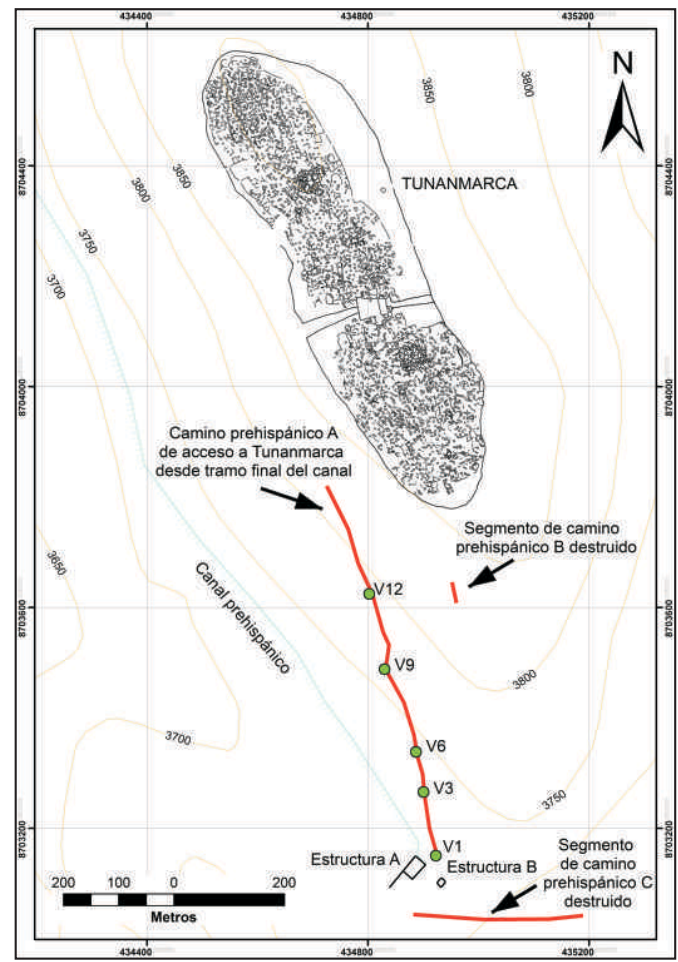

Figura 2: Mapa de ubicación de las evidencias arqueológicas registradas (basado en la Carta Nacional IGN, hoja 24-I, esc. 1:100,000. Intervalo de grilla: 400 metros; esc. de publicación: 1:6,000) 
en la investigación de campo en arqueología (15). Una vez identificadas todas las evidencias a nivel de superficie, se registró su localización mediante una unidad GPS convencional Garmin Etrex, en el sistema de coordenadas UTM con datum horizontal WGS 84 y en referencia al norte verdadero. Paralelamente se efectuó el registro fotográfico correspondiente y se hicieron mediciones con cintas métricas, así como dibujos de sección con el empleo de niveles y plomada, en los puntos donde las evidencias se hallaban en mejor estado de conservación.

Una vez en gabinete la información obtenida con la unidad GPS fue trasladada a una computadora, generándose tablas en Microsoft Excel, desde donde fueron procesadas a fin de obtener archivos conocidos como "shapefiles" que puedan ser proyectados como capas en mapas en ArcGIS, en la proyección UTM con datum WGS 84, la misma que fue almacenada en archivos con extensión .pri (16). Este trabajo fue complementado con la georreferenciación del plano del complejo de Tunanmarca que fue elaborado por el proyecto UMARP en años anteriores, a partir de fotografías aéreas (17). Dicha georreferenciación se hizo en base a datos tomados con nuestra unidad GPS, considerando además la escala y el norte consignados en los planos publicados por los integrantes de dicho proyecto $(10,17)$. Por su parte, los dibujos de sección fueron pasados en limpio para su elaboración final y su respectiva digitalización en archivos con extensión .jpg, en tanto que las fotografías fueron editadas en el programa Microsoft Office Picture Manager a fin de mejorar su calidad.

\section{RESULTADOS}

Los reconocimientos de campo permitieron identificar y registrar de manera íntegra, entre otras cosas, los restos de un antiguo camino que une el extenso complejo de Tunanmarca con el tramo final del canal prehispánico que forma parte del sistema hidráulico cercano a dicho yacimiento arqueológico. Dicho camino, denominado $A$ en nuestro registro (Figura 02), presenta una longitud total de $730 \mathrm{~m}$, estando su extremo inferior a $3735 \mathrm{msnm}$ y a escasos treinta metros al noreste de los restos de una gran estructura de planta rectangular de 35 $m$ de largo y $25 \mathrm{~m}$ de ancho (Estructura $A$ ), cuyos muros, de doble hilera de bloques grandes e irregulares de piedra calcárea, presentan un espesor promedio entre 0,60 y $0,80 \mathrm{~m}$, y una altura promedio de 0,40 m sobre el nivel actual del suelo (Figura 03). Por el oeste dicha estructura, cuyo eje mayor se encuentra orientado hacia el noreste, con un acimut de $48^{\circ}$, se halla asociada a un muro recto que tiene la misma orientación y que presenta en total $37 \mathrm{~m}$ de longitud, estando conformado también por doble hilera de bloques grandes e irregulares de piedra calcárea, y que alcanza a tener en promedio $0,80 \mathrm{~m}$ de espesor y $0,50 \mathrm{~m}$ de altura sobre el nivel actual del suelo. El canal prehispánico asociado a Tunanmarca parece terminar, precisamente, en la gran estructura rectangular que acabamos de describir en este párrafo.

De otra parte, cerca del extremo inferior del camino reportado, a $37 \mathrm{~m}$ al sur, se observan los vestigios de otra estructura que presenta restos de muros de una sola hilera de bloques grandes de piedra calcárea de $0,40 \mathrm{~m}$ de espesor y $0,50 \mathrm{~m}$ de altura (Estructura B). Esta construcción, que también tiene la misma orientación que la descrita anteriormente, es mucho más pequeña, toda vez que cuenta sólo con nueve metros de largo y $8,30 \mathrm{~m}$ de ancho (Figura 03). Asimismo, presenta planta ligeramente rectangular, debido a que hacia la parte central de cada uno de sus lados los muros son rectos, mientras que hacia sus esquinas éstos son curvos. A ello cabe agregar la presencia de una especie de banqueta en el interior de la estructura, hacia su esquina norte, definida por un pequeño muro de contención de bloques pequeños y medianos de roca calcárea, asentada con un tipo de argamasa de barro. Tal posible banqueta presenta un ancho de $1,97 \mathrm{~m}$ y $0,30 \mathrm{~m}$ de altura.

El camino prehispánico $A$ que asciende al 


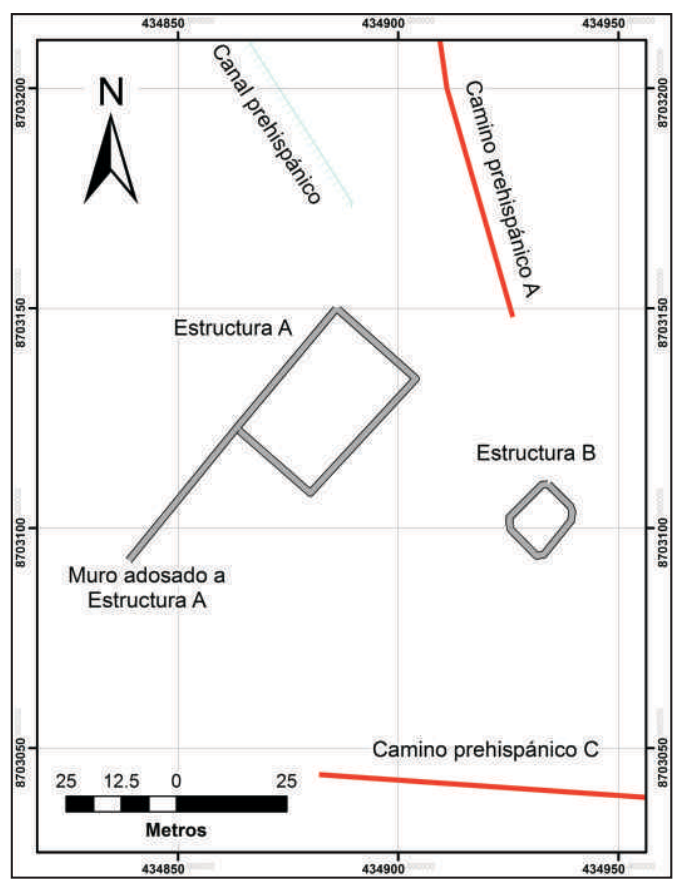

Figura 3: Plano de los elementos y estructuras arqueológicas asociadas al tramo final del canal de Tunanmarca (Intervalo de grilla: 50 metros; esc. de publicación: $1: 1,000)$

complejo de Tunanmarca parte, como dijimos, cerca de las estructuras $A$ y $B$, en las coordenadas UTM 434926E y $8703148 \mathrm{~N}$ (Zona 18L, WGS 84), en donde presenta $1.90 \mathrm{~m}$ de ancho (vértice 01, Figura 04). Desde allí sube por el flanco sur del cerro en dirección norte-noroeste, a través de una ladera de pendiente suave a moderada, con escasa cobertura edáfica y presencia de vegetación silvestre conformada por gramíneas de altura. En los primeros $150 \mathrm{~m}$ el camino mantiene un ancho más o menos constante entre 1,50 y 1,90 m, y una pendiente promedio de $4^{\circ}$. Sin embargo, entre los vértices 03 y 06 se vuelve más estrecho (Foto $01)$, con un metro de ancho en promedio.

Precisamente en dicho tramo la pendiente de la ladera del cerro es más pronunciada y allí el camino se halla en un estado de deterioro más avanzado, aunque pudimos observar que también su propia pendiente se incrementa a poco más de $5^{\circ}$. Más adelante, entre los vértices 06 y 09, hay indicios de posibles ramificaciones del camino, aunque hoy en día son casi imperceptibles por el pésimo estado de preservación de tales evidencias. Estas ramificaciones parecen correr de manera paralela al trazo del camino principal. Entre los vértices 09 y 12 el camino mantiene el ancho promedio de un metro (Foto 02), pero en algunas partes su pendiente sobrepasa los $6^{\circ}$, situación que cambia más arriba, en su tramo superior, al norte del vértice 12, donde la pendiente baja a $3^{\circ}$, aunque el camino mantiene el mismo ancho promedio hasta el último punto correspondiente al vértice 15, en donde pudo ser registrado en forma segura, en las coordenadas UTM $434725 \mathrm{E}$ y $8703820 \mathrm{~N}$ (Zona 18L, WGS 84) y a una altitud de 3825 msnm (Tabla 01).

Tabla 1. Coordenadas UTM y altitud de los vértices correspondientes al camino A (Datum horizontal WGS 84, Zona 18L).

\begin{tabular}{cccc}
\hline Vértice & Este & Norte & Altitud \\
\hline V1 & 434926 & 8703148 & 3735 \\
V2 & 434911 & 8703200 & 3744 \\
V3 & 434902 & 8703262 & 3751 \\
V4 & 434899 & 8703298 & 3755 \\
V5 & 434890 & 8703328 & 3758 \\
V6 & 434889 & 8703334 & 3758 \\
V7 & 434883 & 8703370 & 3762 \\
V8 & 434865 & 8703428 & 3770 \\
V9 & 434832 & 8703488 & 3781 \\
V10 & 434838 & 8703532 & 3786 \\
V11 & 434827 & 8703556 & 3789 \\
V12 & 434808 & 8703624 & 3798 \\
V13 & 434782 & 8703680 & 3808 \\
V14 & 434764 & 8703820 & 3816 \\
V15 & 434725 & & 3825 \\
\hline
\end{tabular}


En términos generales, el camino prehispánico A mantiene las mismas características constructivas a lo largo de toda su extensión registrada. Nuestros dibujos de corte muestran que la preparación de la plataforma del camino se llevó a cabo modificando ligeramente el terreno natural, es decir, cortando muy poco la roca madre que constituye el sustrato geológico calcáreo del cerro, y formando rellenos de tierra de poca altura que se estabilizaron con muros de contención de piedra también calcárea, extraída probablemente del mismo lugar (Figura 04). Dichos muros de contención están conformados por bloques pétreos irregulares medianos y grandes, dispuestos en la mayoría de casos en doble hilera y posiblemente asentados con algún tipo de mezcla de barro que hoy no se observa de modo claro. El espesor que alcanzan estos muros oscila entre los 0,30 y 0,45 m, en tanto que su altura es mucho más variable, entre 0,10 y $0,50 \mathrm{~m}$, siendo notablemente más baja en puntos donde la pendiente natural del terreno es más suave. En última instancia, cabe indicarse la ausencia de

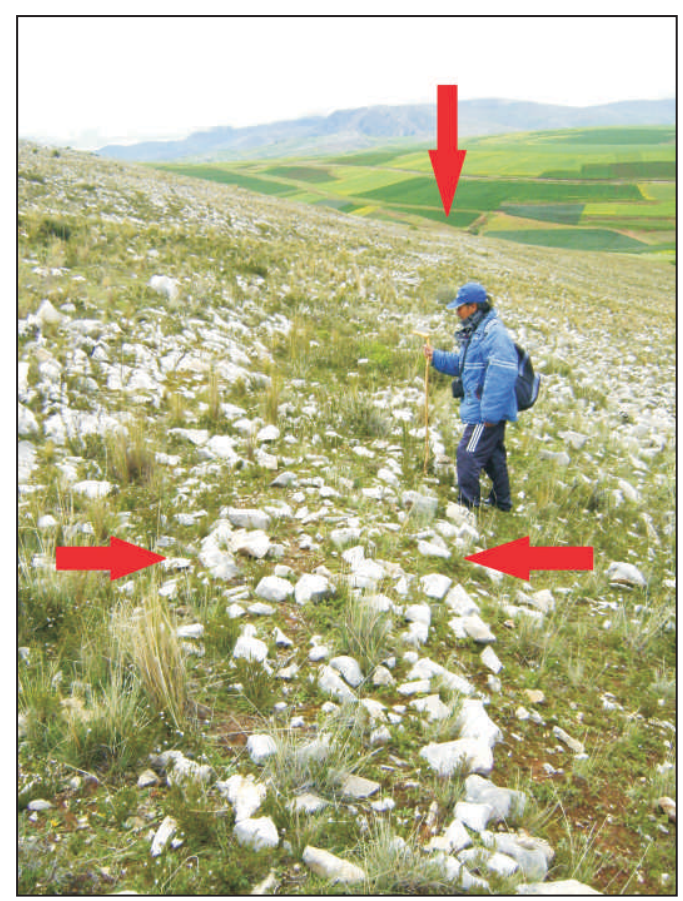

Foto 1: Estado actual del camino A cerca del vértice 06. La flecha vertical señala la ubicación del vértice 01 . Las flechas horizontales indican los bordes del camino. otros rasgos asociados al camino como escalinatas de piedra o canaletas para drenaje de aguas superficiales.

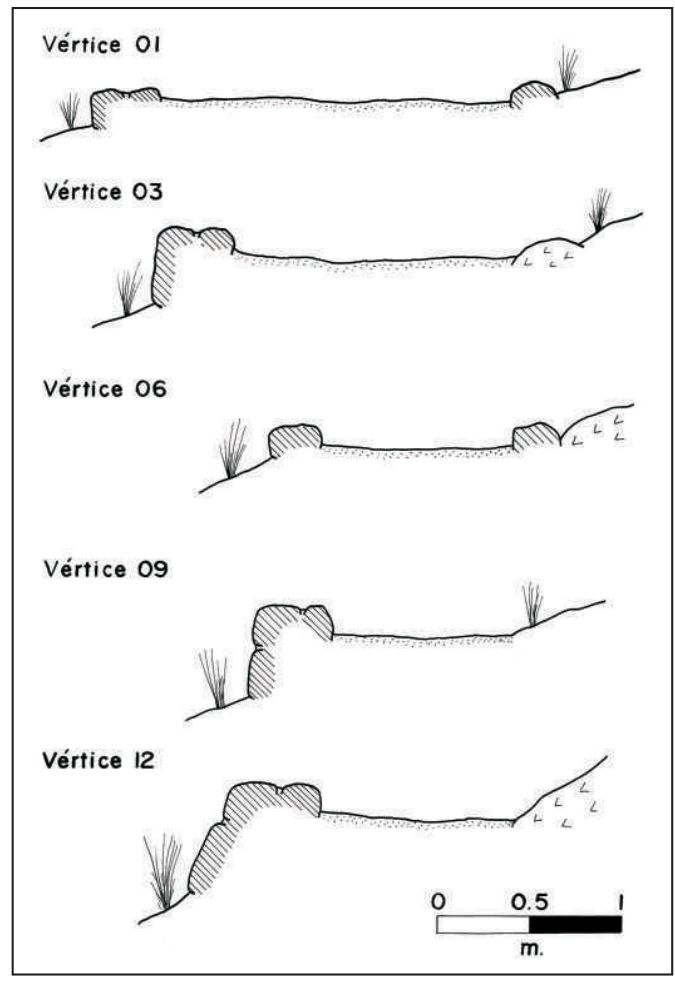

Figura 4: Cortes del camino prehispánico Aen los vértices 01, 03, 06, 09 y 12 (Esc. 1:20. Todos los cortes miran al norte. Los achurados señalan muros de contención y otros rasgos culturales asociados al camino).

A la altura del vértice 12 del camino prehispánico $A$, a unos $140 \mathrm{~m}$ al este, se observan los restos de otro camino, denominado $B$ en nuestro registro, esta vez muy deteriorado y visible únicamente en un tramo de unos $35 \mathrm{~m}$ aproximadamente. Su extremo inferior se ubica en las coordenadas UTM 434960E y $8703608 \mathrm{~N}$ (Zona 18L, WGS 84) y se halla a una altitud de 3840 msnm, en tanto que su extremo superior, próximo a la primera muralla del complejo de Tunanmarca, se encuentra en las coordenadas UTM $434952 \mathrm{E}$ y $8703646 \mathrm{~N}$ (Zona 18L, WGS 84) y a una altitud de 3843 msnm. Este segmento de camino muestra características formales bastante similares al camino prehispánico $A$, aunque su pésimo estado de preservación no permite un registro más detallado.

Finalmente, cabe reportar la presencia de un 
tercer camino de data prehispánica, denominado $\mathrm{C}$ en nuestro registro. $\mathrm{A}$ diferencia de los dos anteriores, este otro camino no asciende a la parte alta del cerro donde se encuentra el yacimiento de Tunanmarca, sino más bien corre por su base meridional (Figura 02). Claramente se le puede observar a lo largo de un tramo de alrededor de $310 \mathrm{~m}$, que se dirige de este a oeste, estando cortado en ambos extremos por campos de cultivo modernos que en la actualidad han borrado el trazo original de dicho camino. Su extremo oriental se encuentra en las coordenadas UTM 435189 E y 8703042 (Zona 18L, WGS 84) y sobre los $3691 \mathrm{msnm}$. En este punto el camino tiene un ancho de alrededor de nueve metros y allí precisamente se observan los restos de una escalinata conformada por un conjunto visible de 16 escalones de piedra asentada con barro, cuyo ancho oscila entre 1,40 y 1,90 m (Foto 03). Por su parte, el extremo occidental del camino se halla en las coordenadas UTM 434882E y $8703044 \mathrm{~N}$ (Zona 18L, WGS 84) y a una altitud de 3730 msnm. Aquí el camino es mucho más angosto, con dos metros de ancho promedio y presenta muros de contención de bloques irregulares grandes y medianos de piedra calcárea de 0,50 m de espesor, los mismos que definen su plataforma y alcanzan hasta unos 0,60 m de altura. Más hacia el oeste de este punto el presente camino se encuentra, como dijimos, destruido en su totalidad por la expansión de la frontera agrícola. Muy cerca, a unos escasos $50 \mathrm{~m}$ al norte, se encuentran las estructuras $A$ y $B$ previamente descritas y que se asocian, como vemos, al tramo final del canal prehispánico de Tunanmarca y al camino prehispánico A (Figura 03).

\section{DISCUSIÓN}

Los hallazgos reportados en esta oportunidad permiten acercarnos un poco más a las formas de organización sociopolítica de las poblaciones establecidas en tiempos prehispánicos en el valle de Yanamarca y en el extenso asentamiento de Tunanmarca. Si bien el canal que forma parte del sistema hidráulico asociado a dicho yacimiento ya había sido reportado en la década de 1970 (13) y estudiado en cierto detalle algunos años después (14), hasta el momento no se tenía, como ya dijimos al principio, conocimiento de indicio alguno que ayudase a establecer una asociación directa entre dicho sistema hidráulico y el complejo arqueológico de Tunanmarca.

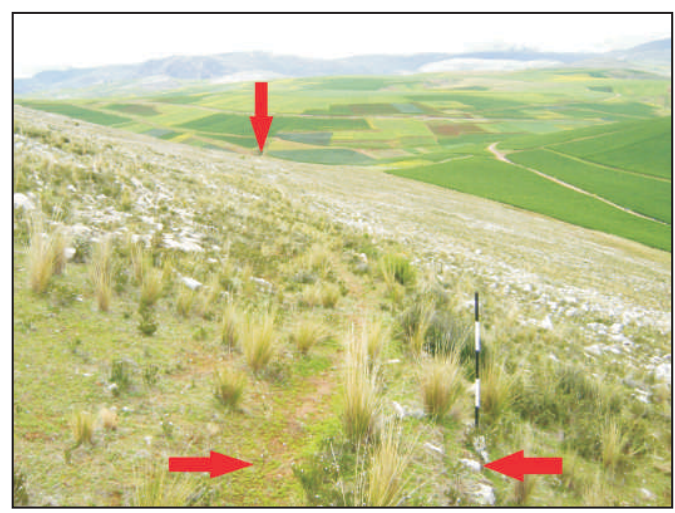

Foto 2: Estado actual del camino A cerca del vértice 09. La flecha vertical señala la ubicación del tramo inicial del camino, entre los vértices 01 y 03 . Las flechas horizontales indican los bordes del camino.

Los autores consideramos que un primer mérito del hallazgo del camino prehispánico A radica precisamente en que ahora tal asociación cuenta con una evidencia material visible. Más aún, un segundo aspecto interesante de dicho camino es que éste presenta características formales similares a otros caminos prehispánicos en la región, además de un trazo que vincula el espacio donde parece terminar el recorrido del canal y la sección central de Tunanmarca, lo cual podría tener importantes implicancias en relación a la naturaleza de la infraestructura arqueológica registrada en el lugar, como veremos a continuación.

Por un lado, resulta curiosa la cercanía espacial entre el tramo final del canal, la Estructura A y el muro recto adosado a esta última. Aunque no hay indicios claros a nivel de la superficie actual del terreno, pensamos que dicha estructura, por sus dimensiones y características, pudo haber constituido una especie de reservorio en donde se habría almacenado un volumen significativo de 
agua traída mediante el canal mencionado. Es probable también que haya existido un reservorio anexo $u$ otro tipo de infraestructura asociada a la Estructura $A$, pero que fue destruido por la expansión de los campos agrícolas modernos y de la cual solo queda el gran muro recto de piedras que se adosa a la estructura señalada por su esquina oeste.

En cuanto a la Estructura B, su función todavía nos resulta enigmática, aunque su ubicación y características nos sugieren que pudo haber estado asociada tanto con el canal, así como con la Estructura $A$ y el camino prehispánico $A$, destacando que este último parece dirigirse en forma directa hacia ella. Tomando en cuenta todo esto, no resulta descabellado pensar que el camino prehispánico A permitía acceder, desde Tunanmarca, a cierto tipo de infraestructura relacionada al almacenaje de agua obtenida mediante el sistema hidráulico en mención.

De otra parte, debemos resaltar el hecho de que el camino prehispánico $A$ parece dirigirse, en su tramo superior, hacia la parte central del asentamiento de Tunanmarca, precisamente en la zona donde se abre un amplio espacio alargado, delimitado por muros perimétricos de piedra, que corre en sentido perpendicular al eje mayor del área ocupada por el asentamiento, dividiéndolo en dos mitades a la altura de donde se hallan las probables "plazas" o espacios públicos de dicho sitio $(9,18)$. Sin embargo, aunque el trazo que sigue el camino prehispánico $A$ es, en este sentido, bastante sugerente, el pésimo estado de conservación en sus tramos superiores finales nos impide saber, con precisión y certeza, hacia qué parte del asentamiento de Tunanmarca se dirige este camino (Figura 02).

Considerando que la ocupación de Tunanmarca se produjo durante la segunda parte del periodo Intermedio Tardío, entre los años 1350 y 1450 d.C. $(9,10)$, ahora es posible asignar la misma antigüedad para el canal, el camino A y las estructuras A y B. Por su emplazamiento y características, esto también se podría pensar para el camino prehispánico $B$, en tanto que la cuestión cronológica con relación al camino prehispánico C resulta más problemática, puesto que esta vía no parece dirigirse a ninguno de los demás rasgos y elementos arqueológicos presentados en este reporte, así como tampoco asciende hacia el sitio de Tunanmarca. Estas observaciones resultan coherentes con el hecho de que el camino C presenta características distintas en relación a los caminos A y B, como presencia de escalinatas de piedra bien definidas y un ancho considerablemente mucho mayor. Evaluando estos aspectos y basándonos en estudios sobre la red vial inca (19), se puede pensar que el camino C podría datar, finalmente, de los tiempos del periodo Horizonte Tardío (1450-1533 d.C.).

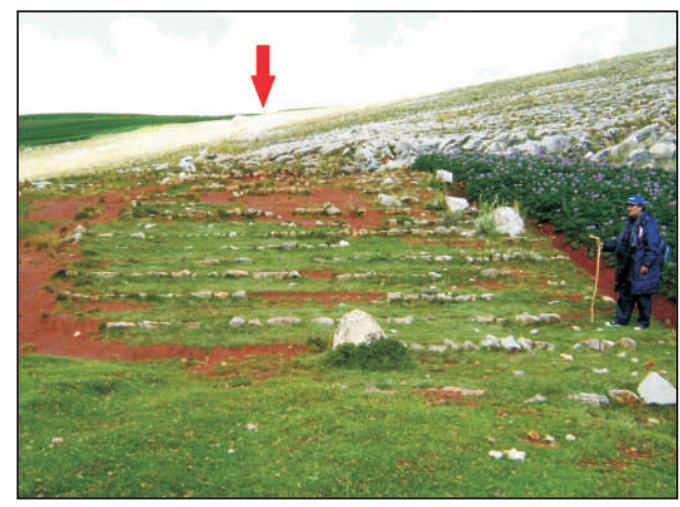

Foto 3: Detalle de las escalinatas en el extremo este del camino C. La flecha señala la ubicación del extremo oeste del tramo conservado y mapeado de dicho camino.

Como ya se dijo al principio, investigaciones previas han llegado a la conclusión que Tunanmarca fue la sede de una organización sociopolítica relativamente compleja de nivel de jefatura, que estuvo integrada, además, por los habitantes de un conjunto de centros poblados y aldeas cercanas quienes, motivados por la situación de profundo faccionalismo que caracterizó a la región durante la segunda mitad del Intermedio Tardío (20), habrían buscado establecer alianzas con los líderes de Tunanmarca $u$ obtener favores de parte de ellos. Dentro de este panorama, se ha dicho que una de las bases del poder político de los líderes de la jefatura de Tunanmarca y de otras en la región habría guardado relación con la economía, debido a su rol protagónico en la organización de la fuerza 
de trabajo de la comunidad para el desarrollo de proyectos orientados al incremento de la productividad agropecuaria de sus tierras, y a su capacidad de toma de decisiones en la distribución de los recursos $(7,9)$. Sin embargo, también se ha afirmado que el desarrollo de la tecnología de riego en el valle de Yanamarca no fue sofisticado durante el Intermedio Tardío, razón por la cual su implementación y manejo pudo efectuarse en un nivel comunitario, sin un gran nivel de especialización, lo que a su vez impidió que ciertos líderes incrementaran su poder basados exclusivamente en el conocimiento sobre hidráulica y afines $(7,14)$.

Todo parece indicar, más bien, que la principal fuente del poder de los líderes de jefaturas como la de Tunanmarca vino de la situación de inseguridad y conflicto intercomunitario que se vivió por aquel tiempo. Habría sido en aquel entonces que ganaron prestigio y poder aquellos que destacaban en conocimientos, destrezas y otras habilidades necesarias para garantizar la integridad de la comunidad y su defensa en caso de alguna agresión externa, tal como confirman los testimonios contenidos en ciertas fuentes históricas tempranas del siglo XVI $(11,12)$.

En este contexto, las evidencias que hemos reportado parecen respaldar algunos de los planteamientos desarrollados por otros académicos $(7,9)$. Así, se observa que la tecnología para almacenar el agua necesaria diariamente para los más de diez mil habitantes de Tunanmarca no parece haber sido sumamente sofisticada. Es muy probable, además, que parte del volumen de agua transportado se haya empleado para irrigar los campos inmediatamente cercanos al asentamiento, tal como fue sugerido antes por Parsons (13).

Si tomamos en cuenta los resultados de otros estudios, podemos pensar entonces que, tanto en la administración del agua para usos domésticos así como para fines agrícolas, los líderes de Tunanmarca debieron intervenir de algún modo. Por otro lado, las restricciones en el acceso al agua por parte de la gente de Tunanmarca no parecen haber sido muy grandes, toda vez que el camino $A$ no presenta indicios que nos hagan pensar ello. Esto podría indicar que, tal como ha planteado Earle (7), el control del acceso al agua fue algo relativamente importante para los líderes de dicho pueblo, pero no lo suficiente como para determinar su ascenso político y consolidarlo frente a toda la comunidad. Sin embargo, aquí más bien valdría la pena considerar el aspecto ideológico, pues como vimos, el camino A parece relacionarse con los espacios centrales o "plazas" de Tunanmarca. Si ello ocurrió, quizás este camino fue una vía empleada por la comunidad -o al menos parte de ella- en momentos importantes en donde sus miembros, incluyendo a sus líderes, se reunían para llevar a cabo algún acto religioso, cívico o festivo, en cuyo marco se pudieron haber desplazado hacia el reservorio de agua u otro tipo de infraestructura asociada a su sistema hidráulico, tal como hoy hacen muchas comunidades rurales andinas contemporáneas (21). Debemos ver si en otros asentamientos contemporáneos de la región se hallan indicios que nos ayuden a evaluar estos planteamientos para, de este modo, conocer un poco mejor la naturaleza del poder que estuvo detrás de la autoridad ejercida por los sinches de las jefaturas del valle de Yanamarca durante la segunda mitad del periodo Intermedio Tardío y así desarrollar contribuciones teóricas sobre las trayectorias de evolución sociopolítica de las jefaturas desde una perspectiva andina. 


\section{REFERENCIAS BIBLIOGRAFICAS}

1. Fried M. The Evolution of Political Society. An Essay in Political Anthropology. New York: Random House; 1967.

2. Carneiro R. A theory on the origin of the state. Science. 1970; 169: 733-738.

3. Service E. A Century of Controversy. Ethnological Issues from 1860 to 1960. Orlando: Academic Press; 1985.

4. Haas J. Editor. From Leaders to Rulers. New York: Kluwer Academic / Plenum Publishers; 2001.

5. Feinman G. Five Points about Power. En: O'Donovan M, editor. The Dynamics of Power. Carbondale: Center for Archaeological Investigations, Southern Illinois University; 2002. pp. 387-393.

6. Earle T. Chiefdoms in archaeological and ethnohistorical perspectives. Annual Review of Anthropology. 1987; 16: 279-308.

7. Earle T. How Chiefs Come to Power. The Political Economy in Prehistory. Stanford: Stanford University Press; 1997.

8. Carneiro R. The chiefdom as precursor of the state. En: Jones G, Kautz R, editores. The Transition to Statehood in the New World. Cambridge: Cambridge University Press; 1981. pp. 37-79.

9. Earle T. The Tunanmarca Polity of Highland Peru and its Settlement System (AD 1350-1450). En: Blanton R, editor. Settlement, Subsistence, and Social Complexity. Essays Honoring the Legacy of Jeffrey R. Parsons. Los Angeles: Cotsen Institute of Archaeology, University of California, Los Angeles; 2005. pp. 89-118.

10.D'Altroy T, Hastorf C. Editores. Empire and Domestic Economy. New York: Kluwer Academic / Plenum Publishers; 2001.

11. Toledo F. Información hecha por Orden de Don Francisco de Toledo en su Visita de las Provincias del Perú, en la que Declaran Indios Ancianos sobre el Derecho de los Caciques y sobre el Gobierno que Tenían Aquellos Pueblos antes que los Incas los Conquistasen. Concepción de Xauxa, 20 de Noviembre 1570. En: Levillier R, editor. Don Francisco de Toledo, supremo organizador del Perú: Su vida, su obra (1515-1582). Buenos Aires: EspasaCalpe; 1940 [1570]. Vol. 2. pp. 14-37.

12. Vega A. La descripcion que se hizo en la provincia de Xauxa por la instruccion de S.M. que á la dicha provincia se invió de molde. En: Rivera E. Imagen de Jauja. Huancayo: Universidad Nacional del Centro del Perú; s/f. pp. 249-266.

13.Parsons J. El complejo hidráulico de Tunanmarca. Canales, acueductos y reservorios. En: Matos R, editor. Actas y Trabajos del III Congreso Pervano El Hombre y la Cultura Andina. Lima: Editora Lasontay; 1978. Tomo II. pp. 556-566.

14. Hastorf C, Earle T. Intensive agriculture and the geography of political change in the Upper Mantaro Region of Central Peru. En: Farrington I, editor. Prehistoric Intensive Agriculture in the 
Tropics. Oxford: British Archaeological Records Series; 1985. pp. 569-597.

15. Banning E. Archaeological Survey. New York: Kluwer Academic / Plenum Publishers; 2002.

16. Conolly J, Lake M. Geographical Information Systems in Archaeology. 1a ed. Cambridge: Cambridge University Press; 2006.

17. Earle T, D'Altroy T, Hastorf C, Scott C, Costin C, Russell G, Sandefur E. Archaeological Field Research in the Upper Mantaro, Peru, 1982-1983: Investigations of Inka Expansion and Exchange. Los Angeles: Institute of Archaeology, University of California, Los Angeles; 1987.

18. DeMarrais E. The architecture and organization of Xauxa settlements. En: D'Altroy T, Hastorf C, editores. Empire and Domestic Economy. New York: Kluwer Academic / Plenum Publishers; 2001. pp. 115-153.

19. Hyslop J. The Inka Road System. Orlando: Academic Press; 1984.

20.D'Altroy T. Factions and political development in the central Andes. En: Brumfiel E, Fox J, editores. Factional Competition and Political Development in the New World. Cambridge: Cambridge University Press; 1994. pp. 171-187.

21.Farfán C. El simbolismo en torno al agua en la comunidad de Huaros-Canta. Boletín del Instituto Francés de Estudios Andinos. 2002; 31 (1): 115-142.

Correo electrónico:

mperales@continental.edu.pe, lacasadelcaminante@hotmail.com 